\title{
Medium-range interactions and crossover to classical critical behavior
}

\author{
Erik Luijten* and Henk W. J. Blöte \\ Department of Physics, Delft University of Technology, P.O. Box 5046, 2600 GA Delft, The Netherlands \\ Kurt Binder \\ Institut für Physik, Johannes Gutenberg Universität, Staudinger Weg 7, D-55128 Mainz, Germany
}

(Received 3 July 1996)

\begin{abstract}
We study the crossover from Ising-like to classical critical behavior as a function of the range $R$ of interactions. The power-law dependence on $R$ of several critical amplitudes is calculated from renormalization theory. The results confirm the predictions of Mon and Binder, which were obtained from phenomenological scaling arguments. In addition, we calculate the range dependence of several corrections to scaling. We have tested the results in Monte Carlo simulations of two-dimensional systems with an extended range of interaction. An efficient Monte Carlo algorithm enabled us to carry out simulations for sufficiently large values of $R$, so that the theoretical predictions could actually be observed. [S1063-651X(96)12810-5]
\end{abstract}

PACS number(s): 64.60.Ak, 05.70.Jk, 64.60.Fr, 75.10.Hk

\section{INTRODUCTION}

As is well known, the critical behavior of a physical system strongly depends on the range of the interactions. The longer the range, the stronger critical fluctuations will be suppressed. In the limit of infinite range we recover classical or mean-field-like critical behavior. For interactions with a finite range, however, fluctuations remain very important and essentially modify the critical behavior. As follows from the Ginzburg criterion [1], sufficiently close to the critical temperature $T_{c}$ nonclassical critical exponents apply for any finite interaction range $R$. This crucial difference between finite and infinite $R$ implies a crossover from one type of critical behavior to another as a function of $R$. Such crossover phenomena are of great interest for a wealth of critical phenomena. They occur, e.g., in polymer mixtures (see Ref. [2] and references therein), as a function of the chain length, and gas-liquid transitions, as a function of the difference between the temperature and the critical temperature. The explanation of these phenomena in terms of competing fixed points of a renormalization transformation is one of the important features of the renormalization theory (see, e.g., Ref. [3]).

In Ref. [4], Mon and Binder have already studied crossover as a function of $R$ within the context of finite-size scaling, motivated by the crossover in polymer mixtures. They predicted that the critical amplitudes of scaling functions display a singular dependence on $R$. The various power-law dependencies were obtained from phenomenological crossover scaling arguments. In this paper, we will derive this dependence on $R$ from a renormalization description of the crossover from classical to nonclassical critical behavior. The first part of the renormalization trajectory is governed by the Gaussian fixed point, which is unstable for $d<4$. The corresponding scaling relations have been derived by

*Electronic address: erik@tntnhb3.tn.tudelft.nl
Rikvold et al. [5]. Sufficiently close to criticality, the final part of the renormalization trajectory is governed by the Ising fixed point. The resulting relations are in complete agreement with the predictions from Ref. [4]. In addition, we obtain the $R$ dependence of the leading corrections to scaling and derive from renormalization arguments a logarithmic factor in the shift of the critical temperature. This factor was already conjectured in Ref. [4].

It is interesting to note that the physical mechanism leading to the singular range dependence of scaling functions is closely related to that leading to the violation of hyperscaling for $d>4$. The latter effect is caused by a singular dependence of thermodynamic quantities on the coefficient $u$ of the $\phi^{4}$ term for $u \rightarrow 0$ in a Landau-Ginzburg-Wilson (LGW) Hamiltonian. In other words, $u$ is a so-called dangerous irrelevant variable (see, e.g., Ref. [6] for a more detailed discussion). Here, as we will see, the fact that this coefficient becomes small for large values of $R$ plays again an essential role, although $u$ is relevant for $d<4$.

Furthermore, we present Monte Carlo results for twodimensional Ising models with an extended range of interaction. A serious problem associated with such simulations is that the simulation time tends to increase rapidly with the number of interacting spins. However, a large interaction range is crucial to observe the predicted $R$ dependencies, as will follow from the renormalization description. The maximum range that could be accessed in Ref. [4] was too small to verify the theoretical predictions. We overcome this limitation by means of an efficient cluster Monte Carlo algorithm [7], in which the simulation time per spin is practically independent of the range of the interactions.

The outline of this paper is as follows. In Sec. II, we derive the $R$ dependence of critical amplitudes from renormalization theory. These results are verified by Monte Carlo simulations, presented in Sec. III. We end with our conclusion in Sec. IV. Two technical issues, namely, the Fourier transform of a spherical distribution of interactions in a general number of dimensions and the application of the cluster 
algorithm to medium-range interactions, are addressed in Appendixes A and B, respectively.

\section{RENORMALIZATION DERIVATION OF THE DEPENDENCE OF CRITICAL AMPLITUDES ON THE INTERACTION RANGE}

A model with long-range interactions which has attracted much attention is that in which the spin-spin interactions decay algebraically as a function of the distance $r$ between the spins, $K(r)=A r^{-d-\sigma}(\sigma>0)$, where $d$ is the dimensionality and $A>0$ the interaction strength (see, e.g., Refs. $[8,9,6])$. For $d<4$, this model displays an interesting continuous variation of critical behavior as function of $\sigma$ : for $0<\sigma \leqslant d / 2$ the critical behavior is classical (mean-field-like), for $d / 2<\sigma<2-\eta_{\mathrm{sr}}$ the critical exponents vary continuously, and for $2-\eta_{\mathrm{sr}}<\sigma$, the interactions decay fast enough to yield short-range (Ising-like) critical behavior. Here, $\eta_{\text {sr }}$ denotes the exponent $\eta$ in the $d$-dimensional model with shortrange interactions. The algebraical decay of the interactions is responsible for the existence of an intermediate regime between Ising-like and classical critical behavior. In this paper we focus on a different way to interpolate between the long-range (mean-field) limit and short-range models. Instead, we choose ferromagnetic interactions which are constant within a range $R$ and zero beyond this range. Thus, we have the following Hamiltonian:

$$
\mathcal{H} / k_{B} T=-\sum_{i} \sum_{j>i} K_{d}\left(\mathbf{r}_{i}-\mathbf{r}_{j}\right) s_{i} s_{j}-h_{0} \sum_{i} s_{i}
$$

where the spin-spin interaction $K_{d}(\mathbf{r}) \equiv c R^{-d}$ for $|\mathbf{r}| \leqslant R$ and the sums run over all spins in the system. This Hamiltonian displays physical behavior that is different from the powerlaw case. In particular, the intermediate regime with variable exponents is absent, and mean-field critical behavior is restricted to the infinite-range limit. We analyze the influence of the range $R$ within the context of renormalization theory, starting from a generalized Landau-Ginzburg-Wilson Hamiltonian, where the $[\nabla \phi(\mathbf{r})]^{2}$ term normally representing the (short-range) interactions is replaced by an interaction term with spin-spin coupling (A1),

$$
\begin{aligned}
\mathcal{H}(\phi) / k_{B} T= & \int_{V} d \mathbf{r}\left\{-\frac{1}{2} \int_{\left|\mathbf{r}-\mathbf{r}^{\prime}\right| \leqslant R} d \mathbf{r}^{\prime}\left[\frac{c}{R^{d}} \phi(\mathbf{r}) \phi\left(\mathbf{r}^{\prime}\right)\right]\right. \\
& \left.-h_{0} \phi(\mathbf{r})+\frac{1}{2} v \phi^{2}(\mathbf{r})+u_{0} \phi^{4}(\mathbf{r})\right\} .
\end{aligned}
$$

As a consequence of the normalization factor $R^{-d}$, the critical value of the temperature parameter $c$ depends only weakly on $R$. The first integral runs over the volume $V$ which contains $N$ particles. We adopt periodic boundary conditions. The Fourier transform of the interaction is calculated in Appendix A. It leads to the following momentum-space representation of the Hamiltonian:

$$
\begin{aligned}
\mathcal{H}\left(\phi_{\mathbf{k}}\right) / k_{B} T= & \frac{1}{2} \sum_{\mathbf{k}}\left[-c\left(\frac{2 \pi}{k R}\right)^{d / 2} J_{d / 2}(k R)+v\right] \phi_{\mathbf{k}} \boldsymbol{\phi}_{-\mathbf{k}} \\
& +\frac{u_{0}}{4 N} \sum_{\mathbf{k}_{1}} \sum_{\mathbf{k}_{2}} \sum_{\mathbf{k}_{3}} \phi_{\mathbf{k}_{1}} \phi_{\mathbf{k}_{2}} \phi_{\mathbf{k}_{3}} \phi_{-\mathbf{k}_{1}-\mathbf{k}_{2}-\mathbf{k}_{3}} \\
& -h_{0} \sqrt{\frac{N}{2}} \phi_{\mathbf{k}=\mathbf{0}} .
\end{aligned}
$$

$J_{\nu}$ is a Bessel function of the first kind of order $\nu$. The wave vectors are discrete because of the periodic boundary conditions. Furthermore, we restrict the wave vectors to lie within the first Brillouin zone, which is reminiscent of the underlying lattice structure. The interaction term can be expanded in a Taylor series containing only even terms in $k R$. This means that we will be mainly concerned with the term of order $(k R)^{2}$, because higher-order terms will turn out to be irrelevant. The constant term in the Taylor series is absorbed in $\bar{v}$ and the coefficient of the quadratic term as a factor in $\bar{c}$. This yields a new Hamiltonian,

$$
\begin{aligned}
& \mathcal{H}_{t}\left(\phi_{\mathbf{k}}\right) / k_{B} T=\frac{1}{2} \sum_{\mathbf{k}}\left[\overline{c R^{2}} k^{2}+\vec{v}\right] \phi_{\mathbf{k}} \phi_{-\mathbf{k}} \\
& +\frac{u_{0}}{4 N} \sum_{\mathbf{k}_{1}} \sum_{\mathbf{k}_{2}} \sum_{\mathbf{k}_{3}} \phi_{\mathbf{k}_{1}} \phi_{\mathbf{k}_{2}} \phi_{\mathbf{k}_{3}} \phi_{-\mathbf{k}_{1}-\mathbf{k}_{2}-\mathbf{k}_{3}} \\
& -h_{0} \sqrt{\frac{N}{2}} \phi_{\mathbf{k}=\mathbf{0}} \text {. }
\end{aligned}
$$

Since we are free to choose the scale on which the fluctuations of the order parameter are measured, we may rescale $\phi \rightarrow \psi \equiv \sqrt{\bar{c}} R \phi$. This is generally the most convenient choice because the dominant $k$-dependent term becomes independent of $R$. (Naturally, this rescaling is not compulsory and the same results will be obtained without it, provided one keeps track of the dependence of the nontrivial fixed point on $\bar{c} R^{2}$, arising from the integration over propagators in the inner part of the Brillouin zone.) This leads to

$$
\begin{aligned}
\widetilde{\mathcal{H}}\left(\psi_{\mathbf{k}}\right) / k_{B} T= & \frac{1}{2} \sum_{\mathbf{k}}\left[k^{2}+\frac{\bar{v}}{\bar{c} R^{2}}\right] \psi_{\mathbf{k}} \psi_{-\mathbf{k}} \\
& +\frac{u_{0}}{4 \bar{c}^{2} R^{4} N} \sum_{\mathbf{k}_{1}} \sum_{\mathbf{k}_{2}} \sum_{\mathbf{k}_{3}} \psi_{\mathbf{k}_{1}} \psi_{\mathbf{k}_{2}} \psi_{\mathbf{k}_{3}} \psi_{-\mathbf{k}_{1}-\mathbf{k}_{2}-\mathbf{k}_{3}} \\
& -\frac{h_{0}}{\sqrt{\bar{c} R}} \sqrt{\frac{N}{2}} \psi_{\mathbf{k}=\mathbf{0}} .
\end{aligned}
$$

The parameter $\bar{c}$ is merely a constant, independent of the range, and in order not to be hampered by it in the future analysis, we absorb the various powers of it in $r_{0} \equiv \bar{v} / \bar{c}$, $u \equiv u_{0} / \vec{c}^{2}$, and $h \equiv h_{0} / \sqrt{\bar{c}}$. Now, $r_{0}$ assumes the role of the temperature parameter.

If the range $R$ is large, the coefficient of the $\psi^{4}$ term is relatively small and hence the critical behavior of the system is determined by the Gaussian fixed point. Under a renormalization transformation with a rescaling parameter $l$ the Hamiltonian thus transforms as 


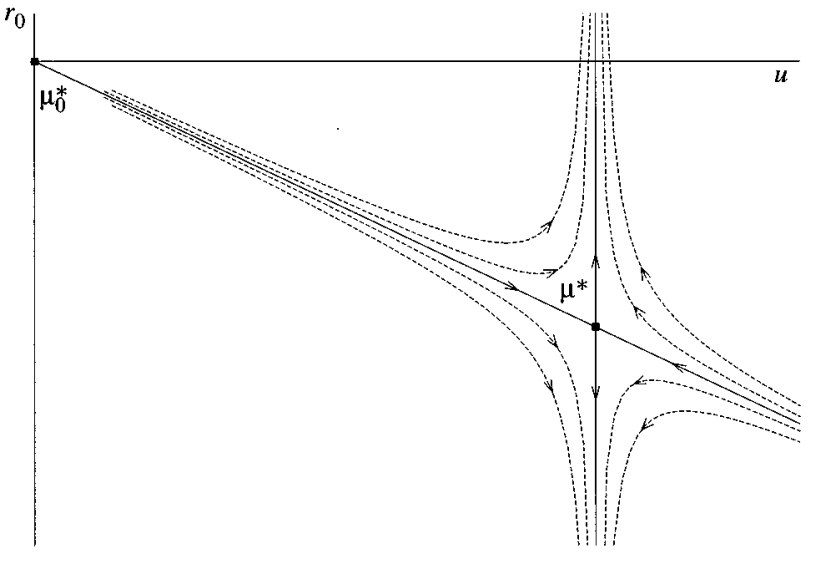

FIG. 1. Qualitative picture of the renormalization trajectory describing the crossover from the Gaussian fixed point $\mu_{0}^{*}=(0,0)$ to the Ising fixed point $\mu^{*}=\left(r_{0}^{*}, u^{*}\right)$.

$$
\begin{aligned}
\left.\widetilde{\mathcal{H}^{\prime}(} \psi_{\mathbf{k}^{\prime}}^{\prime}\right) / k_{B} T^{\prime}= & \frac{1}{2} \sum_{\mathbf{k}^{\prime}}\left[k^{\prime 2}+\frac{r_{0}}{R^{2}} l^{2}\right] \psi_{\mathbf{k}^{\prime}}^{\prime} \psi_{-\mathbf{k}^{\prime}}^{\prime}+\frac{u}{4 R^{4} N^{\prime}} l^{4-d} \\
& \times \sum_{\mathbf{k}_{1}^{\prime}} \sum_{\mathbf{k}_{2}^{\prime}} \sum_{\mathbf{k}_{3}^{\prime}} \psi_{\mathbf{k}_{1}^{\prime}}^{\prime} \psi_{\mathbf{k}_{2}^{\prime}}^{\prime} \psi_{\mathbf{k}_{3}^{\prime}}^{\prime} \psi_{-\mathbf{k}_{1}^{\prime}-\mathbf{k}_{2}^{\prime}-\mathbf{k}_{3}^{\prime}}^{\prime} \\
& -\frac{h}{R} \sqrt{\frac{N^{\prime}}{2}} l^{1+d / 2} \psi_{\mathbf{k}^{\prime}=\mathbf{0}}^{\prime} .
\end{aligned}
$$

Here $\psi_{\mathbf{k}^{\prime}}^{\prime}=l^{-1} \psi_{\mathbf{k}}, \mathbf{k}^{\prime}=\mathbf{k} l$, the sums run again over the full Brillouin zone, and $N^{\prime}=N l^{-d}$. For $d<4$, the $\psi^{4}$ term grows and the system moves away from the Gaussian fixed point $\mu_{0}^{*}$ (see Fig. 1). At present, we are interested in the flow from the neighborhood of the Gaussian fixed point to that of the Ising fixed point. Thus we remain close to the critical line connecting the two fixed points and the temperature field parametrized by $r_{0}$ remains small. The crossover to Isinglike critical behavior occurs when the coefficient of the $\psi^{4}$ term is of the same order as that of the $k^{2} \psi^{2}$ term, which is unity, i.e., when $l=l_{0} \equiv R^{4 /(4-d)}$. We shall refer to $l_{0}$ as the crossover scale.

By comparing the coefficient of the $\psi^{4}$ term to that of the $r_{0} \psi^{2}$ term, it is possible to derive a criterion that states for which temperatures the critical behavior will be Ising-like and for which temperatures it will be classical. This is the well-known Ginzburg criterion [1], which can also be derived from Eq. (6) (see, e.g., Ref. [10], p. 107). One expects the Gaussian fixed point to dominate the renormalization flow if, irrespective of $l$, the $\psi^{4}$ coefficient is small compared to the temperature coefficient. Thus, one requires the scaled combination $u R^{-4} l^{4-d} /\left(r_{0} R^{-2} l^{2}\right)^{(4-d) / 2}$ to be small, or $r_{0}^{(4-d) / 2} R^{d} u^{-1} \gg 1$ [cf. also Ref. [4], Eq. (3)].

Since we are now in the neighborhood of the Ising fixed point, we continue renormalizing our Hamiltonian with nonclassical renormalization exponents $y_{t}, y_{h}$, and $y_{i}$. To leading order, it will transform as follows, where $b$ denotes the rescaling factor of our new transformation:

$$
\begin{aligned}
\widetilde{\mathcal{H}}^{\prime \prime}\left(\psi_{\mathbf{k}^{\prime \prime}}^{\prime \prime}\right) / k_{B} T^{\prime \prime}= & \frac{1}{2} \sum_{\mathbf{k}^{\prime \prime}}\left[k^{\prime \prime 2}+R^{2 d /(4-d)}\left(b^{y_{t}} \widetilde{r_{0}}+r_{0}^{*}\right)\right] \psi_{\mathbf{k}^{\prime \prime}}^{\prime \prime} \psi_{-\mathbf{k}^{\prime \prime}}^{\prime \prime} \\
& +\frac{b^{y_{i}} \widetilde{u}+u^{*}}{4 N^{\prime \prime}} \\
& \times \sum_{\mathbf{k}_{1}^{\prime \prime}} \sum_{\mathbf{k}_{2}^{\prime \prime}} \sum_{\mathbf{k}_{3}^{\prime \prime}} \psi_{\mathbf{k}_{1}^{\prime \prime}}^{\prime \prime} \psi_{\mathbf{k}_{2}^{\prime \prime}}^{\prime \prime} \psi_{\mathbf{k}_{3}^{\prime \prime}}^{\prime \prime} \psi_{-\mathbf{k}_{1}^{\prime \prime}-\mathbf{k}_{2}^{\prime \prime}-\mathbf{k}_{3}^{\prime \prime}}^{\prime \prime} \\
& -h R^{3 d /(4-d)} \sqrt{\frac{N^{\prime \prime}}{2}} b^{y_{h}} \psi_{\mathbf{k}^{\prime \prime}=\mathbf{0}}^{\prime \prime} .
\end{aligned}
$$

We have introduced the coefficients $\widetilde{r_{0}}$ and $\tilde{u}$, which denote the location of the point $\left(r_{0}, u\right)$ in the new coordinates with respect to the nontrivial (Ising) fixed point $\mu^{*}$ which we are now approaching (see Fig. 1).

The singular part of the free energy density, $f_{s}$, is after the transformation $\phi \rightarrow \psi$ denoted by $\widetilde{f_{s}}$,

$$
f_{s}\left(r_{0}, u, h\right)=\tilde{f}_{s}\left(\frac{r_{0}}{R^{2}}, \frac{u}{R^{4}}, \frac{h}{R}\right) .
$$

Furthermore, we introduce the quantity $\hat{f}_{s}\left(\widetilde{r_{0}}, \tilde{u}, h\right)$ $\equiv \widetilde{f}_{s}\left(r_{0}, u, h\right)$. Because the total free energy is conserved along the renormalization trajectory, the singular part of the free energy density changes as

$$
\begin{aligned}
\widetilde{f}_{s}( & \left.\frac{r_{0}}{R^{2}}, \frac{u}{R^{4}}, \frac{h}{R}\right) \\
& =l^{-d} \widetilde{f}_{s}\left(\frac{r_{0}}{R^{2}} l^{2}, \frac{u}{R^{4}} l^{4-d}, \frac{h}{R} l^{1+d / 2}\right) \\
& =R^{-4 d /(4-d)} \hat{f}_{s}\left({\widetilde{r_{0}}}^{2 d /(4-d)}, \tilde{u}, h R^{3 d /(4-d)}\right) \\
& =b^{-d} R^{-4 d /(4-d)} \hat{f}_{s}\left(t R^{2 d /(4-d)} b^{y_{t}}, \widetilde{u} b^{y_{i}}, h R^{3 d /(4-d)} b^{\left.y_{h}\right)},\right.
\end{aligned}
$$

where we have used the notation $t \equiv\left[T-T_{c}(R)\right] / T_{c}(R)$ for $\widetilde{r_{0}}$. In Fig. 1, $t$ stands for the distance to the critical line connecting $\mu_{0}^{*}$ and $\mu^{*}$. In the second equality we have substituted the crossover scale, $l=R^{4 /(4-d)}$. Of course, this is only a qualitative measure for the location of the crossover, but the renormalization predictions for the scaling exponents are exact. The relation (9), which holds for $1<d<4$, is the key to the scaling relations obtained on phenomenological grounds in Ref. [4]. We will first illustrate this by deriving the $R$ dependence of the critical amplitudes of the magnetization density $m$ and the magnetic susceptibility $\chi$. The magnetization density can be calculated by taking the first derivative of the free energy density with respect to the magnetic scaling field $h$,

$$
\begin{aligned}
m= & \frac{\partial f_{s}}{\partial h}\left(r_{0}, u, h\right) \\
= & \frac{\partial \widetilde{f}_{s}}{\partial h}\left(\frac{r_{0}}{R^{2}}, \frac{u}{R^{4}}, \frac{h}{R}\right) \\
= & b^{y_{h}-d} R^{-d /(4-d)} \\
& \times \hat{f}_{s}^{(1)}\left(t R^{2 d /(4-d)} b^{y_{t}}, \widetilde{u} b^{y_{i}}, h R^{3 d /(4-d)} b^{\left.y_{h}\right),}\right.
\end{aligned}
$$


where $\hat{f}_{s}^{(1)}$ denotes the first derivative of $\hat{f}_{s}$ with respect to its third argument. Here we have neglected any nonlinear relation between the magnetic scaling field $h$ and the physical magnetic field. Furthermore, we have omitted a contribution from the analytic part of the free energy, because $h$ only couples to the $\mathbf{k}=\mathbf{0}$ (uniform) mode. To extract the dependence of $m$ on $t$ and $R$ from Eq. (10), we choose the rescaling factor $b$ such that the first argument of the derivative of $\hat{f}_{s}$ is equal to 1 , i.e., $b=t^{-1 / y_{t}} R^{-2 d /\left[y_{t}(4-d)\right]}$, and set the irrelevant variable $\tilde{u}$ and the magnetic scaling field $h$ equal to zero,

$$
m=t^{\beta} R^{(2 d \beta-d) /(4-d)} \hat{f}_{s}^{(1)}(1,0,0) .
$$

This result agrees with Ref. [4], Eq. (34). In the same way we can calculate the magnetic susceptibility from $\hat{f}_{s}$ by taking the second derivative with respect to $h$,

$$
\begin{aligned}
\chi & =\frac{\partial^{2} f_{s}}{\partial h^{2}}\left(r_{0}, u, h\right) \\
& =\frac{\partial^{2} \widetilde{f}_{s}}{\partial h^{2}}\left(\frac{r_{0}}{R^{2}}, \frac{u}{R^{4}}, \frac{h}{R}\right) \\
& =b^{2 y_{h}-d} R^{2 d /(4-d)} \hat{f}_{s}^{(2)}\left(t R^{2 d /(4-d)} b^{y_{t}}, \widetilde{u} b^{y_{i}}, h R^{3 d /(4-d)} b^{y_{h}}\right) .
\end{aligned}
$$

Choosing the arguments of the second derivative of $\hat{f}_{s}$ as in Eq. (11), we find

$$
\chi=t^{-\gamma} R^{2 d(1-\gamma) /(4-d)} \hat{f}_{s}^{(2)}(1,0,0),
$$

in agreement with Ref. [4], Eq. (39). In Eqs. (11) and (13) we have used the well-known relations between the renormalization exponents and the critical exponents (see, e.g., Table 18 in Ref. [11]).

The finite-size scaling behavior of thermodynamic functions can also be derived from this renormalization scenario by including a finite-size field $1 / L$ as an additional argument of the free energy density in Eq. (9). Under the first renormalization transformation this scaling field will scale as $l / L$ and under the second renormalization transformation as $l b / L=b R^{4 /(4-d)} L^{-1}$. The finite-size scaling behavior is found by choosing $b$ such that $l b / L=1$, i.e., $b=L R^{-4 /(4-d)}$. Substituting this into Eqs. (10) and (12), we obtain for $m$

and for $\chi$,

$$
m=L^{y_{h}-d} R^{\left(3 d-4 y_{h}\right) /(4-d)} \hat{f}_{s}^{(1)}\left(t L^{y_{t}} R^{-2\left(2 y_{t}-d\right) /(4-d)}, \widetilde{u} L^{y_{i}} R^{-4 y_{i} /(4-d)}, h L^{y_{h}} R^{\left(3 d-4 y_{h}\right) /(4-d)}\right),
$$

and for $\chi$,

$$
\chi=L^{2 y_{h}-d} R^{2\left(3 d-4 y_{h}\right) /(4-d)} \hat{f}_{s}^{(2)}\left(t L^{y_{t}} R^{-2\left(2 y_{t}-d\right) /(4-d)}, \widetilde{u} L^{y_{i}} R^{-4 y_{i} /(4-d)}, h L^{y_{h}} R^{\left(3 d-4 y_{h}\right) /(4-d)}\right) .
$$

These results agree with Ref. [4], where the prefactors of the magnetization density and the magnetic susceptibility were predicted as, respectively, $L^{-\beta / \nu} R^{(2 \beta-\gamma) /[\nu(4-d)]}$ and $L^{\gamma / \nu} R^{(4 \beta-2 \gamma) /[\nu(4-d)]}$. Furthermore, the first argument of the scaling functions was predicted as $t L^{1 / \nu} R^{\mathcal{K}}$, with $\mathcal{K}=-(2 \alpha) /[\nu(4-d)]$ [Ref. [4], Eq. (25)]. This is indeed equivalent with our result $\mathcal{K}=-2\left(2 y_{t}-d\right) /(4-d)$. However, the predicted range dependence of the critical amplitudes [i.e., of the prefactors in Eqs. (14) and (15)] is only valid in the limit of infinite range. For smaller ranges, $R$-dependent correction terms are present. These correction terms can be calculated as well. They do not come from the dependence of the scaling functions on the irrelevant fields, as corrections to scaling normally do: these corrections vanish in the thermodynamic limit. However, they come from higher-order contributions to the renormalization of the $\psi^{4}$ coefficient which were previously neglected in the derivation of the crossover scale $l_{0}$. Note that in the neighborhood of the Gaussian fixed point, the terms $\psi^{n}$ with $n<2 d /(d-2)$ are relevant and that for $d=2$ all higher-order terms are equally relevant. However, the coefficients of these terms are, after the rescaling $\phi \rightarrow \psi$, proportional to $R^{-n}$, so the leading contribution comes from the term $w R^{-6} \psi^{6}$. Under a spatial rescaling with a factor $l=e^{s}$, the renormalization equation for this term is, to leading order,

$$
\frac{d w^{\prime}}{d s}=(6-2 d) w^{\prime} .
$$

The solution of this equation, $w^{\prime}(s)=w e^{(6-2 d) s}$, can be substituted in the renormalization equation for the $\psi^{4}$ coefficient,

$$
\frac{1}{R^{4}} \frac{d u^{\prime}}{d s}=(4-d) \frac{u^{\prime}}{R^{4}}+a \frac{w^{\prime}}{R^{6}} .
$$

To first order in $w$, this yields

$$
\begin{aligned}
\frac{u^{\prime}}{R^{4}} & =e^{(4-d) s} \frac{1}{R^{4}}\left[u+\frac{a}{2-d} \frac{w}{R^{2}}\left(e^{(2-d) s}-1\right)\right] \\
& =l^{4-d} \frac{1}{R^{4}}\left[u+\frac{a}{2-d} \frac{w}{R^{2}}\left(l^{2-d}-1\right)\right],
\end{aligned}
$$

where $u$ and $w$ denote the values of $u^{\prime}$ and $w^{\prime}$ at $l=1$, respectively. This implies that the previously obtained crossover scale $l_{0}=R^{4 /(4-d)}$ is multiplied by a factor $\left(1+\widetilde{a} R^{-2}\right)$ and hence all critical amplitudes will exhibit this correction. However, the solution (18) is not valid for $d=2$, where $u R^{-4} \psi^{4}$ and $w R^{-6} \psi^{6}$ are equally relevant. The solution of Eq. (17) is then given by

$$
\frac{u^{\prime}}{R^{4}}=e^{2 s} \frac{1}{R^{4}}\left(u+a \frac{w}{R^{2}} s\right)=l^{2} \frac{1}{R^{4}}\left(u+a \frac{w}{R^{2}} \ln l\right),
$$


which yields a (leading) correction factor $\left[1+R^{-2}\left(\widetilde{a_{1}}\right.\right.$ $\left.\left.+\widetilde{a}_{2} \ln R\right)\right]$ in the crossover scale and the critical amplitudes.

From a similar mechanism we can derive the $R$ dependence of the so-called shift of the critical temperature [[4], Eq. (15)]. A detailed treatment of the shift of $T_{c}$ can be found in, e.g., Refs. [12,6]. It arises from the $u$-dependent contribution in the renormalization equation for the $\psi^{2}$ term,

$$
\frac{1}{R^{2}} \frac{d r_{0}^{\prime}}{d s}=2 \frac{r_{0}^{\prime}}{R^{2}}+c \frac{u^{\prime}}{R^{4}} .
$$

Thus, the first argument on the right-hand side of the first equation in (9) should be replaced by

$$
\begin{aligned}
\frac{r_{0}^{\prime}}{R^{2}} & =l^{2}\left[\left(\frac{r_{0}}{R^{2}}-\widetilde{c} \frac{u_{0}}{R^{4}}\right)+\widetilde{c} \frac{u_{0}}{R^{4}} l^{2-d}\right] \\
& =l^{2} \frac{1}{R^{2}}\left[\left(r_{0}-\widetilde{c} \frac{u_{0}}{R^{2}}\right)+\widetilde{c} \frac{u_{0}}{R^{2}} l^{2-d}\right] .
\end{aligned}
$$

The term between round brackets is proportional to the reduced temperature and the last term is the leading shift. Substitution of the crossover scale $l_{0}$ shows that this shift in the reduced temperature is proportional to $R^{-2 d /(4-d)}$, which indeed vanishes in the mean-field $(R \rightarrow \infty)$ limit. Remarkably, this disagrees with Ref. [4], where a shift $\propto R^{-d}$ was predicted. Unfortunately, it is not possible to settle this issue at present by means of Monte Carlo data, because only results for $d=2$ are available, which is a special case. Namely, for $d=2$ we obtain instead of Eq. (21) the following solution of Eq. (20):

$$
\frac{r_{0}^{\prime}}{R^{2}}=l^{2}\left(\frac{r_{0}}{R^{2}}+c \frac{u_{0}}{R^{4}} \ln l\right)=l^{2} \frac{1}{R^{2}}\left(r_{0}+c \frac{u_{0}}{R^{2}} \ln l\right) .
$$

Thus, we find, upon substitution of the crossover scale, that the shift in the reduced temperature has the form $(p+q \ln R) / R^{2}$, where the constant $p$ comes from a multiplicative factor introduced by the crossover criterion. In Ref. [4], $d=2$ was already suggested as a special case, with possibly logarithmic corrections. The renormalization argument shows that such a $\ln R$ term is indeed present.

Now, let us return to Eq. (4), where we omitted quartic (and higher) terms in $k R$. It follows from the renormalization scenario that terms proportional to $k^{2 n}$ transform as $k^{2 n} l^{2-2 n}$ under the first renormalization transformation and hence are irrelevant for $n>1$. The behavior of these terms under the second renormalization transformation is less simple, but again quartic and higher terms do not influence the leading terms; see, e.g., Ref. [13], Sec. VII.6.

Besides, it can be seen that the structure of the interaction term does not depend on the details of the spin-spin interaction. E.g., replacing the interaction term (A1) with $K(\mathbf{r})=c R^{-d} \exp \left[-(r / R)^{2}\right]$ leads to precisely the same structure of the LGW Hamiltonian and hence to the same scaling relations involving $R$. This is in agreement with the universality hypothesis.

Furthermore, the renormalization description explains why the interaction range $R$ must be large to observe the predicted powers of $R$ in the critical amplitudes: only for systems with $R$ large the renormalization trajectory starts in the neighborhood of the Gaussian fixed point and hence only these systems will accurately display the corresponding $R$
TABLE I. The range of interaction $R_{m}$, the corresponding number of neighbors $z$, and effective range of interaction $R$.

\begin{tabular}{ccc}
\hline \hline$R_{m}^{2}$ & $z$ & $R^{2}$ \\
\hline 2 & 8 & $\frac{3}{2}$ \\
4 & 12 & $\frac{7}{3}$ \\
6 & 20 & $\frac{17}{5}$ \\
8 & 24 & $\frac{25}{6}$ \\
10 & 36 & 6 \\
18 & 60 & $\frac{148}{15}$ \\
32 & 100 & $\frac{81}{5}$ \\
50 & 160 & $\frac{517}{20}$ \\
72 & 224 & $\frac{1007}{28}$ \\
100 & 316 & $\frac{4003}{79}$ \\
140 & 436 & $\frac{7594}{109}$ \\
\hline
\end{tabular}

dependence. Finally, in the finite-size scaling description, the system size must be sufficiently large in order to observe the crossover to Ising-like critical behavior: we require that the rescaling factor $b$ is minimal of order unity, or $L=O\left(R^{4 /(4-d)}\right)$.

\section{MONTE CARLO RESULTS AND COMPARISON WITH THE THEORETICAL PREDICTIONS}

\section{A. Definition of the model}

We have carried out Monte Carlo simulations for twodimensional Ising systems consisting of $L \times L$ lattice sites with periodic boundary conditions and an extended range of interaction. Each spin interacts equally with its $z$ neighbors lying within a distance $R_{m}$, as defined in Eqs. (1) and (A1) with $R$ replaced by $R_{m}$ and $d=2$. The Monte Carlo simulations were carried out using a special cluster algorithm for long-range interactions [7]. Its application to the interactions defined above is described in Appendix B. Following Ref. [4] we define the effective range of interaction $R$ as

$$
\begin{aligned}
R^{2} & \equiv \frac{\sum_{j \neq i}\left(\mathbf{r}_{i}-\mathbf{r}_{j}\right)^{2} K_{i j}}{\sum_{j \neq i} K_{i j}} \\
& =\frac{1}{z} \sum_{j \neq i}\left|\mathbf{r}_{i}-\mathbf{r}_{j}\right|^{2} \quad \text { with }\left|\mathbf{r}_{i}-\mathbf{r}_{j}\right| \leqslant R_{m} .
\end{aligned}
$$

Table I lists the values of $R_{m}^{2}$ for which we have carried out simulations, as well as the corresponding values of $R^{2}$. The ratio between $R^{2}$ and $R_{m}^{2}$ approaches $1 / 2$, as can be simply found when the sums in Eq. (23) are replaced by integrals. Note that the results for $R_{m}^{2}=18$ and $R_{m}^{2}=32$ cannot be compared to those presented by Mon and Binder, because in Ref. [4] the interactions were for these two system sizes spatially distributed within a square, as can be seen from the number of interacting neighbors and the corresponding effective ranges of interaction.

\section{B. Determination of the critical temperature}

The critical temperatures $T_{c}$ of these systems have been determined using the well-known universal amplitude ratio 


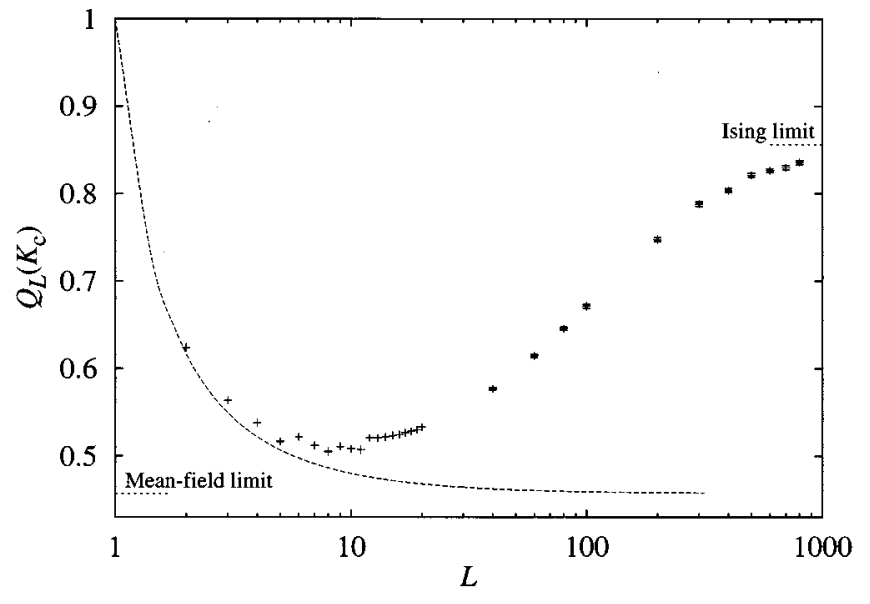

FIG. 2. The critical-point amplitude ratio $Q_{L}\left(K_{c}\right)$ for $R_{m}^{2}=140$ as function of the system size (discrete points). For large $L$, $Q_{L}\left(K_{c}\right)$ approaches the Ising limit $Q_{I} \approx 0.856216$ (dotted line). For decreasing $L, Q_{L}\left(K_{c}\right)$ approaches the mean-field limit $Q_{\mathrm{MF}} \approx 0.456947$ (dotted line), until the system size becomes smaller than the range $R_{m}$ and strong finite-size effects come into play. To illustrate that the system is indeed mean-field-like for these system sizes, we have also plotted $Q$ for finite systems in which all spins interact equally strong (dashed curve). The points for $R_{m}^{2}=140$ indeed approach this curve.

$Q_{L} \equiv\left\langle m^{2}\right\rangle_{L}^{2} /\left\langle m^{4}\right\rangle_{L}$. Both in the Ising and in the mean-field limit the critical-point value of this quantity is accurately known. In the mean-field limit, $Q=Q_{\mathrm{MF}}=8 \pi^{2}$ / $\left[\Gamma\left(\frac{1}{4}\right)\right]^{4} \approx 0.456947$; see Refs. [12] and [7], Appendix A. In the Ising limit, $Q=Q_{I}=0.856216$ (1) [14].

As was noted in Sec. II and also in Ref. [4], rather large system sizes $\left[O\left(R^{2}\right)\right]$ are required to determine $T_{c}$, since $Q$ must approach $Q_{I}$. For $R_{m}^{2} \leqslant 10$ we have included linear system sizes up to $L=500$ and for larger ranges we have used system sizes up to $L=700$ or even $L=800$ $\left(R_{m}^{2}=100,140\right)$. For each run we have generated $10^{6}$ Wolff clusters after equilibration of the system. The various thermodynamic quantities were sampled after every tenth Wolff cluster. In Fig. 2, $Q_{L}\left(K_{c}\right)$ for $R_{m}^{2}=140$ is plotted as function of the system size. One clearly observes the crossover from $Q_{\mathrm{MF}}$ (for $L \ll R_{m}^{2}$ ) to $Q_{I}$ (for $L \gg R_{m}^{2}$ ).

In the Ising limit, the finite-size expansion of $Q_{L}$ reads (see, e.g., Ref. [11])

$$
\begin{aligned}
Q_{L}(K)= & Q+a_{1}\left(K-K_{c}\right) L^{y_{t}}+a_{2}\left(K-K_{c}\right)^{2} L^{2 y_{t}} \\
& +\cdots+b_{1} L^{y_{i}}+b_{2} L^{y_{2}}+\cdots .
\end{aligned}
$$

$K$ denotes the spin-spin coupling, $K_{c}$ the critical coupling, and the $a_{i}$ and $b_{i}$ are nonuniversal (range-dependent) coefficients. The term proportional to $L^{y_{2}}$, with $y_{2}=d-2 y_{h}$, comes from the field dependence of the analytic part of the free energy. In a $\phi^{4}$ theory this term is absent, as was stated in Sec. II, but in a discrete model it should be allowed for. The exponents $y_{t}, y_{h}$, and $y_{i}$, which have already been introduced in the previous section, are, respectively, the temperature, magnetic, and leading irrelevant exponent for the two-dimensional Ising model; $y_{t}=1, y_{h}=\frac{15}{8}$, and $y_{i}=-2$. Table II displays the results of a least-squares fit according to Eq. (25), where $y_{t}, y_{i}$, and $y_{2}$ were kept fixed at their theoretical values. For comparison we have included the estimates for $K_{c}$ from Ref. [4]. Except for $R_{m}^{2}=10$ there is good agreement between the respective estimates. The discrepancy for $R_{m}^{2}=10$ may be explained by the limited range of system sizes in Ref. [4]. Furthermore, for $R_{m}^{2}=2$, which corresponds to the Ising model with nearest and next-nearest neighbor interactions, an accurate transfer-matrix estimate of the critical coupling exists, $K_{c}=0.190192$ 69(5) [15]. The Monte Carlo result agrees with this value. The results for $Q$ are in good agreement with the expected value $Q_{I}$, which confirms not only that universality is satisfied, but also that the maximum system sizes in our simulations are sufficiently large, so that crossover to Ising-like critical behavior indeed has taken place, as it should for an accurate determination of $K_{c}$. In fact, the error margins on $K_{c}$ can be reduced significantly by fixing $Q$ at its Ising value in Eq. (25) (see Table II). Figure 3 illustrates the shift in $1 /\left(z K_{c}\right) \propto T_{\mathrm{c}}$ as function of $R^{-2}$. Even close the mean-field limit $\left(R^{-2} \rightarrow 0\right)$, the deviation of $1 /\left(z K_{c}\right)$ from 1 seems not truly linear. Therefore we have tried to identify the logarithmic term, which was sug-

TABLE II. The amplitude ratio $Q$ and critical coupling $K_{c}$ for the various ranges of interaction studied in this paper. The numbers in parentheses denote the errors in the last decimal places. The fourth column shows the estimates for $K_{c}$ obtained with $Q$ fixed at its Ising value. For comparison, we also list the estimates for $K_{c}$ given in Ref. [4].

\begin{tabular}{rllll}
\hline \hline$R_{m}^{2}$ & \multicolumn{1}{c}{$Q$} & \multicolumn{1}{c}{$K_{c}$} & \multicolumn{1}{c}{$K_{c}$} & $K_{c}$ (Ref. [4]) \\
\hline 2 & $0.8556(5)$ & $0.1901908(19)$ & $0.1901931(11)$ & 0.190 \\
4 & $0.8557(9)$ & $0.1140216(18)$ & $0.1140225(7)$ & 0.11402 \\
6 & $0.8553(7)$ & $0.0631917(8)$ & $0.0631926(4)$ & \\
8 & $0.8553(13)$ & $0.0510460(10)$ & $0.0510467(4)$ & 0.05106 \\
10 & $0.8563(9)$ & $0.0324136(5)$ & $0.03241352(18)$ & 0.032463 \\
18 & $0.8555(14)$ & $0.0185335(3)$ & $0.01853367(9)$ & \\
32 & $0.853(3)$ & $0.01075152(25)$ & $0.01075182(7)$ & \\
50 & $0.856(6)$ & $0.00657274(26)$ & $0.00657276(5)$ & \\
72 & $0.854(4)$ & $0.00464056(16)$ & $0.00464064(4)$ & \\
100 & $0.850(8)$ & $0.00325903(15)$ & $0.00325905(5)$ & \\
140 & $0.862(17)$ & $0.00234637(19)$ & $0.00234631(2)$ & \\
\hline \hline
\end{tabular}




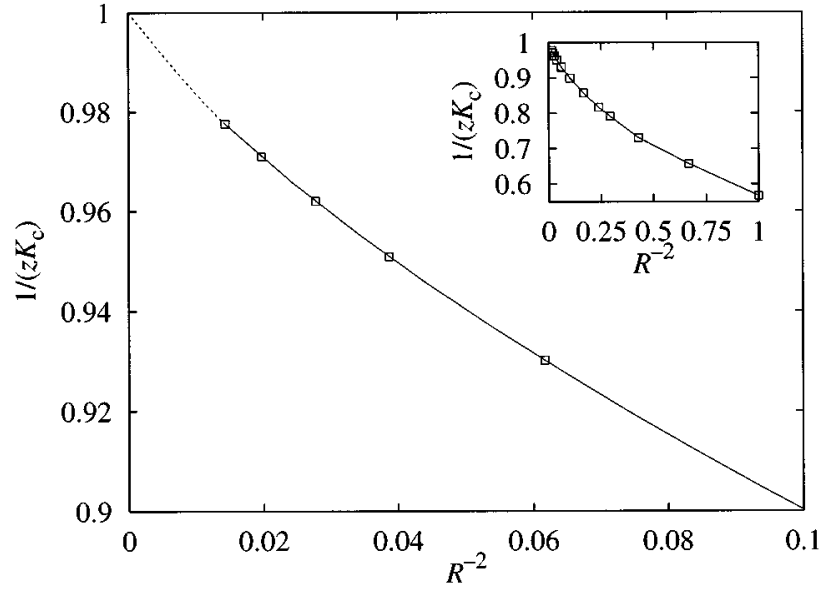

FIG. 3. Plot of $1 /\left(z K_{c}\right)$ versus $R^{-2}$. The dashed line denotes the extrapolation to the mean-field limit. The inset shows $1 /\left(z K_{c}\right)$ over the full range of $R^{-2}$ between the Ising and the mean-field limit.

gested in Ref. [4] and derived from the renormalization scenario in Sec. II, by writing the following expression for the critical coupling,

$$
z K_{c}=1+\frac{p+q \ln R}{R^{2}}
$$

In Fig. 4 we have plotted $\Delta \equiv\left(z K_{c}-1\right) R^{2}$ versus $\ln R$. Indeed, for large values of $R$ the points lie approximately on a straight line, confirming the presence of the logarithmic correction.

Another $\ln R$ correction was suspected in Ref. [4] in the temperature-dependent argument of the finite-size scaling functions. This argument is proportional to $R^{-2\left(2 y_{t}-d\right) /(4-d)}=R^{-2 \alpha /[\nu(4-d)]}$; see Eqs. (14) and (15). For $d=2, \alpha=0$ implies a logarithmic divergence of the specific heat and hence one might expect a similar logarithmic factor here. On the other hand, we have not found a mechanism in the renormalization scenario which could explain such a fac-

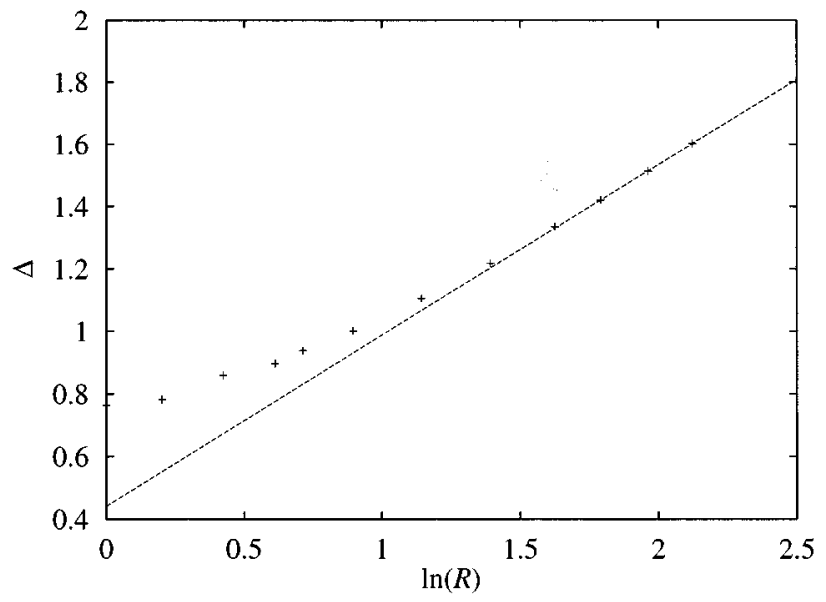

FIG. 4. $\Delta \equiv\left(z K_{c}-1\right) R^{2}$ versus $\ln R$. For large $R$ the graph strongly suggests the presence of a logarithmic correction in the shift of the critical temperature. The error bars do not exceed the symbol size.

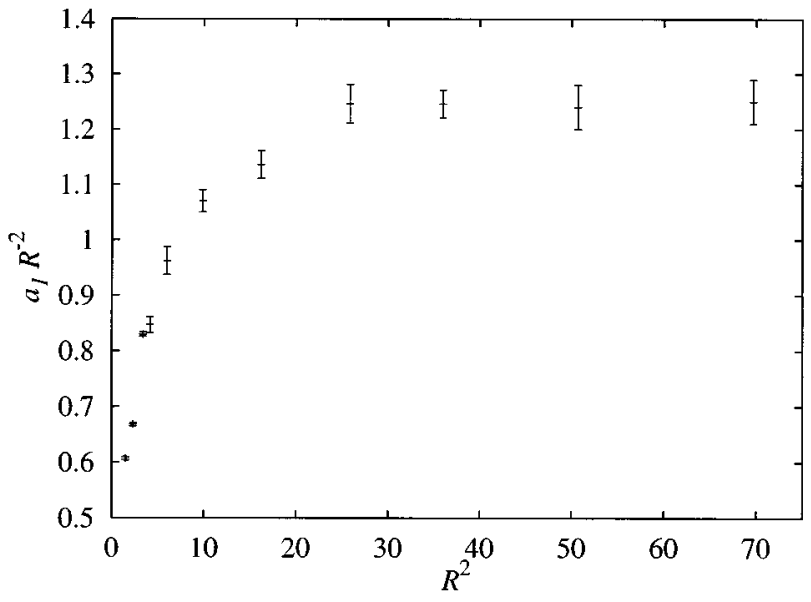

FIG. 5. Range dependence of the amplitude of the temperaturedependent argument of the finite-size scaling function of the universal amplitude ratio $Q$.

tor. Therefore, we have numerically examined the range dependence of the coefficient $a_{1}$ in Eq. (25). Since $\left(K-K_{c}\right)$ is proportional to $R^{-2}$, we must first divide $a_{1}$ by $R^{2}$. Figure 5 displays this quantity as function of the range. For small ranges, there is a strong dependence on $R$, but the coefficients seem to approach a constant value in the large-range limit. This suggests that a logarithmic correction factor is absent.

\section{Range dependence of the magnetization density}

We have sampled the absolute magnetization density, $\langle|m|\rangle$, for which the range dependence is given by Eq. (14). This quantity has been fitted to the following finite-size expansion,

$$
\begin{aligned}
m_{L}(K, R)= & L^{y_{h}-d}\left\{d_{0}(R)+d_{1}(R)\left[K-K_{c}(R)\right] L^{y_{t}}\right. \\
& +d_{2}(R)\left[K-K_{c}(R)\right]^{2} L^{2 y_{t}}+\cdots \\
& \left.+e_{1}(R) L^{y_{i}}+\cdots\right\},
\end{aligned}
$$

where we now have explicitly indicated the range dependence of the parameters. The critical couplings found from this quantity agree well with those obtained from the amplitude ratio $Q$ and the exponent $y_{h}$, listed in Table III, is in good agreement with the exact value 15/8. Furthermore, we have made a least-squares fit with $K_{c}$ fixed at the most accurate values obtained from $Q$. The corresponding estimates for $y_{h}$ are also shown in Table III. They lie even closer to $15 / 8$, which again corroborates that all systems belong to the Ising universality class. From the critical amplitudes $d_{0}(R)$ we can derive the leading $R$ dependence of the magnetization. To increase the accuracy, the values in Table III were determined with $y_{h}$ fixed at its theoretical value. As can be seen from the log-log plot in Fig. 6, the approach to the asymptotic scaling behavior is very slow. Therefore we have determined the scaling exponent in two different ways. A straight line through the points for the three largest ranges yielded $d_{0}(R) \propto R^{-0.738(13)}$, in agreement with the predicted exponent $-3 / 4$ [Eq. (14)]. Inclusion of the correction factor 
TABLE III. The exponent $y_{h}$ and the critical amplitude of the magnetization $d_{0}(R)$ for the various ranges of interaction. The third column shows the estimates for $y_{h}$ obtained with $K_{c}$ fixed at the most accurate values shown in Table II.

\begin{tabular}{rlll}
\hline \hline$R_{m}^{2}$ & \multicolumn{1}{c}{$y_{h}$} & \multicolumn{1}{c}{$y_{h}$} & \multicolumn{1}{c}{$d_{0}(R)$} \\
\hline 2 & $1.8745(7)$ & $1.8749(3)$ & $0.9533(4)$ \\
4 & $1.8763(15)$ & $1.8756(4)$ & $0.8706(5)$ \\
6 & $1.873(3)$ & $1.8767(13)$ & $0.7937(10)$ \\
8 & $1.873(3)$ & $1.8754(8)$ & $0.7523(7)$ \\
10 & $1.874(2)$ & $1.8748(7)$ & $0.6783(6)$ \\
18 & $1.871(3)$ & $1.8740(12)$ & $0.5816(6)$ \\
32 & $1.875(6)$ & $1.8744(9)$ & $0.4929(11)$ \\
50 & $1.873(7)$ & $1.876(2)$ & $0.4181(18)$ \\
72 & $1.865(5)$ & $1.8752(16)$ & $0.3742(8)$ \\
100 & $1.867(9)$ & $1.877(2)$ & $0.3296(8)$ \\
140 & $1.895(13)$ & $1.879(3)$ & $0.2938(13)$ \\
\hline \hline
\end{tabular}

$\left[1+R^{-2}\left(\widetilde{a_{1}}+\widetilde{a_{2}} \ln R\right)\right]$, as predicted from Eq. (19), allowed us to include all data points in the fit and yielded $d_{0}(R)$ $\propto R^{-0.756(5)}$, also in good agreement with the predicted exponent.

\section{Range dependence of the susceptibility}

The magnetic susceptibility can be calculated from the average square magnetization,

$$
\chi=L^{d}\left\langle m^{2}\right\rangle .
$$

We thus expect the following finite-size scaling behavior:

$$
\begin{aligned}
\chi_{L}(K, R)= & s_{0}+L^{2 y_{h}-d}\left\{p_{0}(R)+p_{1}(R)\left[K-K_{c}(R)\right] L^{y_{t}}\right. \\
& +p_{2}(R)\left[K-K_{c}(R)\right]^{2} L^{2 y_{t}}+\cdots \\
& \left.+q_{1}(R) L^{y_{i}}+\cdots\right\} .
\end{aligned}
$$

The term $s_{0}$ comes from the analytic part of the free energy. Because it tends to interfere with the term proportional to $q_{1}(R)$, we have ignored it in the further analysis. Again, the

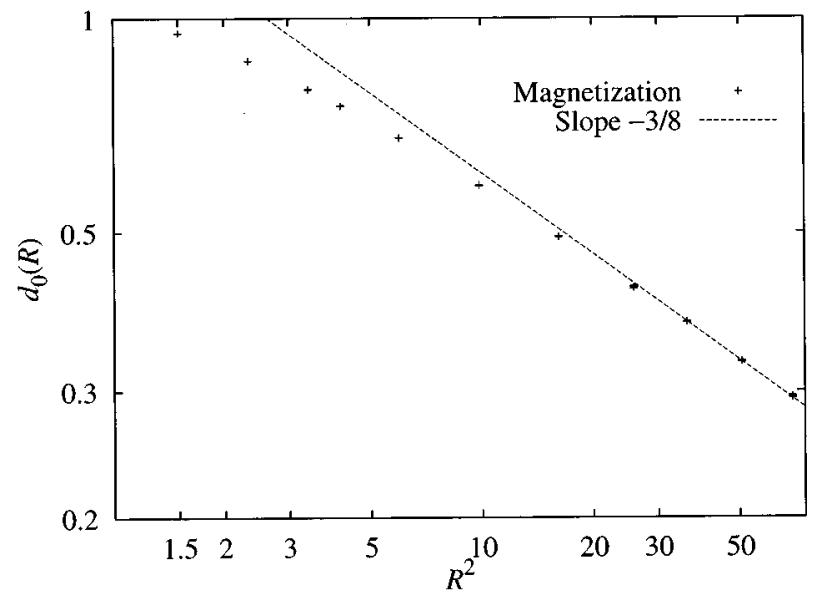

FIG. 6. The critical amplitude $d_{0}(R)$ of the magnetization density versus $R^{2}$.
TABLE IV. The exponent $y_{h}$ and the critical amplitude of the magnetic susceptibility $p_{0}(R)$ for the various ranges of interaction. The third column shows the estimates for $y_{h}$ obtained with $K_{c}$ fixed at the most accurate values shown in Table II.

\begin{tabular}{rlll}
\hline \hline$R_{m}^{2}$ & \multicolumn{1}{c}{$y_{h}$} & \multicolumn{1}{c}{$y_{h}$} & \multicolumn{1}{c}{$p_{0}(R)$} \\
\hline 2 & $1.8754(9)$ & $1.8748(2)$ & $0.9743(9)$ \\
4 & $1.8753(12)$ & $1.8752(3)$ & $0.8136(7)$ \\
6 & $1.8740(18)$ & $1.8761(10)$ & $0.6762(14)$ \\
8 & $1.873(2)$ & $1.8750(6)$ & $0.6076(9)$ \\
10 & $1.874(3)$ & $1.8741(6)$ & $0.4943(7)$ \\
18 & $1.874(4)$ & $1.8740(11)$ & $0.3620(9)$ \\
32 & $1.868(4)$ & $1.873(2)$ & $0.2622(9)$ \\
50 & $1.862(6)$ & $1.874(3)$ & $0.1914(7)$ \\
72 & $1.863(17)$ & $1.870(4)$ & $0.1534(8)$ \\
100 & $1.870(6)$ & $1.874(4)$ & $0.1180(8)$ \\
140 & $1.86(3)$ & $1.870(5)$ & $0.0954(9)$ \\
\hline \hline
\end{tabular}

critical couplings obtained from a least-squares fit lie close to those in Table II and the estimates for $y_{h}$ agree with the Ising value (see Table IV). By repeating the fits with $K_{c}$ fixed at the most accurately known values, the values for $y_{h}$ lie even closer to 15/8 (third column of Table IV). From the parameter $p_{0}(R)$, plotted in Fig. 7, we can extract the leading range dependence of the susceptibility. A straight line through the amplitudes for the three largest ranges gave $p_{0}(R)$ $\propto R^{-1.46(3)}$. For a curve (including the first correction term) through the amplitudes it was necessary to include the data for all ranges $R^{2} \geqslant 7 / 3$ in the fit, in order to determine the coefficient of the $\ln R$ factor. This yielded $p_{0}(R) \propto R^{-1.47(2)}$. Both exponents are in good agreement with the predicted value $2\left(3 d-4 y_{h}\right) /(4-d)=-3 / 2$.

\section{E. Spin-spin correlation function}

The finite-size scaling behavior of the spin-spin correlation function $g(\mathbf{r})$ closely resembles that of the magnetic susceptibility $\chi$, as may be expected from the fact that $\chi$ is the spatial integral of $g$. We also expect the range depen-

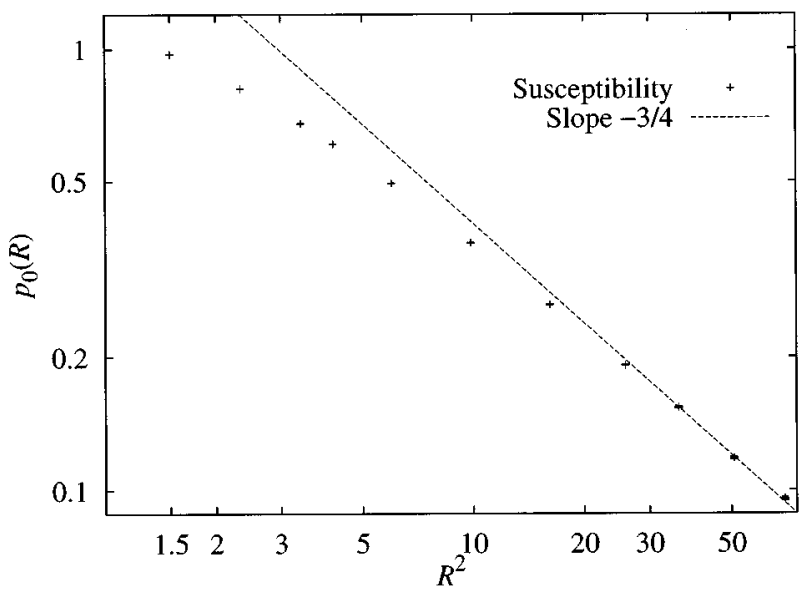

FIG. 7. The critical amplitude $p_{0}(R)$ of the magnetic suscetibility versus $R^{2}$. 
TABLE V. The exponent $y_{h}$ and the critical amplitude of the spin-spin correlation function $v_{0}(R)$ for the various ranges of interaction. The third column shows the estimates for $y_{h}$ obtained with $K_{c}$ fixed at the most accurate values shown in Table II.

\begin{tabular}{rlll}
\hline \hline$R_{m}^{2}$ & \multicolumn{1}{c}{$y_{h}$} & \multicolumn{1}{c}{$y_{h}$} & \multicolumn{1}{c}{$v_{0}(R)$} \\
\hline 2 & $1.8759(8)$ & $1.8754(3)$ & $0.9405(9)$ \\
4 & $1.8744(12)$ & $1.8750(3)$ & $0.7860(6)$ \\
6 & $1.8748(19)$ & $1.8765(11)$ & $0.6528(15)$ \\
8 & $1.8746(17)$ & $1.8754(6)$ & $0.5862(9)$ \\
10 & $1.875(3)$ & $1.8741(7)$ & $0.4770(9)$ \\
18 & $1.874(4)$ & $1.8745(10)$ & $0.3489(9)$ \\
32 & $1.873(4)$ & $1.8747(18)$ & $0.2541(6)$ \\
50 & $1.864(7)$ & $1.876(3)$ & $0.1827(9)$ \\
72 & $1.860(8)$ & $1.871(4)$ & $0.1473(9)$ \\
100 & $1.872(9)$ & $1.876(4)$ & $0.1128(9)$ \\
140 & $1.86(3)$ & $1.871(3)$ & $0.0915(9)$ \\
\hline
\end{tabular}

dence of the two quantities to be the same. We have sampled the correlation function over half the system size and analyzed it using the expansion

$$
\begin{aligned}
g_{L}(K, R)= & L^{2 y_{h}-2 d}\left\{v_{0}(R)+v_{1}(R)\left[K-K_{c}(R)\right] L^{y_{t}}\right. \\
& +v_{2}(R)\left[K-K_{c}(R)\right]^{2} L^{2 y_{t}}+\ldots \\
& \left.+w_{1}(R) L^{y_{i}}+\cdots\right\} .
\end{aligned}
$$

The constant term in (29) is not present here (see, e.g., Ref. [11]). Table $\mathrm{V}$ shows the results for $y_{h}$, both with $K_{c}$ free and fixed. In the latter case, $y_{h}$ is in accurate agreement with its theoretical value, just as for the magnetization density and the magnetic susceptibility. Figure 8 shows a log-log plot of the critical amplitude $v_{0}(R)$ as function of the range. A fit of a straight line through the points with $R^{2}>35$ (i.e., $R_{m}^{2} \geqslant 72$ ) yielded $v_{0}(R) \propto R^{-1.46(3)}$, whereas a curve through all points with $R^{2}>7 / 3$ gave $v_{0}(R) \propto R^{-1.49(2)}$. Both estimates are again in good agreement with the predicted exponent $-3 / 2$.

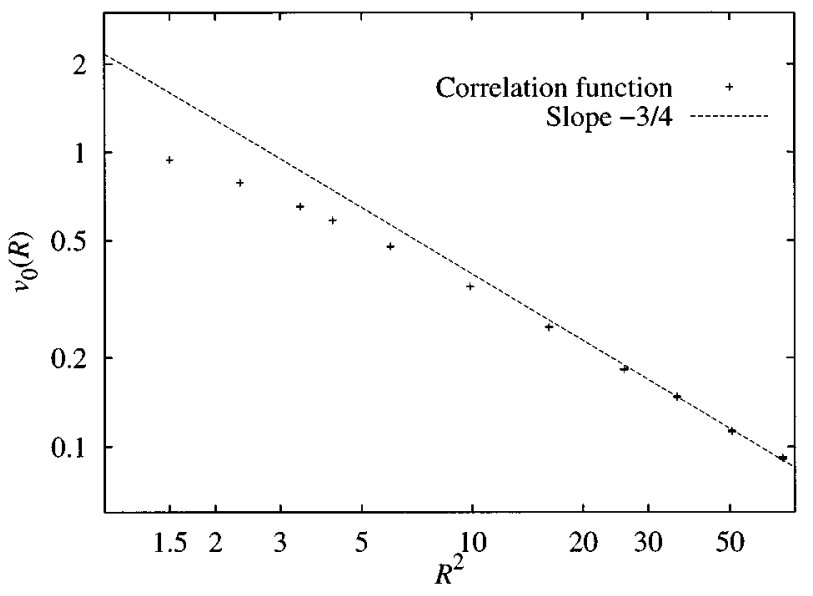

FIG. 8. The critical amplitude $v_{0}(R)$ of the spin-spin correlation function versus $R^{2}$.

\section{CONCLUSION}

In this paper, we have derived the dependence of scaling functions on the range of interactions from renormalizationgroup arguments. The results agree with the predictions of Mon and Binder and yield in addition the corrections to the leading scaling behavior, including the previously conjectured logarithmic factor in the shift of the critical temperature of two-dimensional systems.

We have also carried out accurate Monte Carlo simulations for systems in which the range of the interactions was large enough to verify the theoretical predictions. It was confirmed with high precision that all examined systems belong to the $2 \mathrm{D}$ Ising universality class. Besides the range dependence of critical amplitudes, we also observed the predicted range dependence of the corrections to scaling.

\section{APPENDIX A: FOURIER TRANSFORM OF A SPHERICALLY SHAPED INTERACTION PROFILE}

We define the following isotropic spin-spin interaction $K_{d}$ (the subscript $d$ denotes the dimensionality):

$$
K_{d}(\mathbf{r}) \equiv \begin{cases}c R^{-d} & \text { if }|\mathbf{r}| \leqslant R \\ 0 & \text { if }|\mathbf{r}|>R .\end{cases}
$$

We have normalized the interaction strength, to make the integrated interaction (energy) independent of the range. In this appendix, we calculate the Fourier transform of this interaction for a general number of dimensions. For $d=1$ the calculation is trivial:

$$
\widetilde{K}_{1}(k)=\frac{c}{R} \int_{-R}^{+R} d x e^{i k x}=\frac{2 c}{k R} \sin (k R)
$$

For $d=2$ and $d=3$ one obtains Bessel functions. Using the equality $J_{1 / 2}(x)=\sqrt{2 /(\pi x)} \sin (x)$, the results for $d=1,2,3$ can be summarized as

$$
\widetilde{K}_{d}(\mathbf{k})=c\left(\frac{2 \pi}{k R}\right)^{d / 2} J_{d / 2}(k R),
$$

where $J_{\nu}$ is a Bessel function of the first kind of order $\nu$. This suggests that this equality is valid for general $d$, which can indeed be shown by induction. If we assign the $x$ coordinate to the $(d+1)$ th spatial dimension and use the notation $k_{d}^{2}=\sum_{j=1}^{d} k_{j}^{2}$ we may write

$$
\begin{aligned}
\widetilde{K}_{d+1}(\mathbf{k})= & \frac{c}{R^{d+1}} \int_{-R}^{+R} d x \cos \left(k_{x} x\right)\left(\frac{2 \pi}{k_{d}}\right)^{d / 2}\left(R^{2}-x^{2}\right)^{d / 4} \\
& \times J_{d / 2}\left(k_{d} \sqrt{R^{2}-x^{2}}\right) \\
= & \frac{2 c}{R^{d+1}}\left(\frac{2 \pi}{k_{d}}\right)^{d / 2} \int_{0}^{R} d p \cos \left(k_{x} \sqrt{R^{2}-p^{2}}\right) \\
& \times \frac{p^{(d+2) / 2}}{\sqrt{R^{2}-p^{2}}} J_{d / 2}\left(k_{d} p\right) \\
= & c\left(\frac{2 \pi}{k R}\right)^{(d+1) / 2} J_{(d+1) / 2}(k R)
\end{aligned}
$$


where we have used a Hankel transform of general order; see, e.g., Ref. [16], p. 40, Eq. (48).

\section{APPENDIX B: MONTE CARLO ALGORITHM FOR SPIN SYSTEMS WITH MEDIUM-RANGE INTERACTIONS}

The cluster algorithm we have used for the present Monte Carlo simulations has essentially been described in Ref. [7]. However, that description is rather concise; here, we present a somewhat more elaborate discussion of the mathematical backgrounds, and we outline how the algorithm is applied to medium-range models of the type studied in this paper.

The description is given in terms of the Wolff cluster algorithm [17], but the principle applies only to the cluster formation process. Thus, it is also applicable in the Swendsen-Wang case [18]. For simplicity, we describe the way a cluster of spins is built in the case that there are only ferromagnetic interactions.

For each spin in the cluster, we have to run a task described below. During this task, new spins may be included in the cluster. For this reason, it is convenient to use a "stack" memory containing the addresses of the spins for which the task remains to be done.

The task for a spin $s_{i}$ read from the stack is the following. A loop is executed over all neighbors $s_{j}$ interacting with $s_{i}$. In each step of this loop, the bond connecting sites $i$ and $j$ is "activated" with a probability

$$
p\left(s_{i}, s_{j}\right)=\delta_{s_{i} s_{j}} p,
$$

where $p \equiv 1-\exp (-2 K)$, in which $K$ is the coupling between $s_{i}$ and $s_{j}$. The simulation process would conventionally include a test whether $s_{i}$ and $s_{j}$ are parallel, and if so, the production of a uniformly distributed pseudorandom number $r$. If $r<p$, the bond is activated, $s_{j}$ is added to the cluster, and its address $j$ is stored in the stack memory. Since this loop runs over all neighbors interacting with $s_{i}$, i.e., over all sites within a distance $R_{m}$, the process becomes very time consuming when the range $R_{m}$ of the interactions becomes large, just as in the case of Metropolis simulations.

However, the cluster formation process can be formulated in a more efficient way. Part of the work involved in the activation of the bonds between $s_{i}$ and its neighbors $s_{j}$ can be done in a way that does not depend on the signs of the spins. Thus, as a first step, the bonds connected to $s_{i}$ are "provisionally activated" with a probability $p$, independent of their relative sign. Typically, only a small number of bonds will be provisionally activated for each entry $i$ in the stack memory (i.e., each spin in the cluster). Then, in the second step, the provisionally active bonds, say between sites $i$ and $j$, are actually activated if $s_{i}=s_{j}$, i.e., with probability $\delta_{s_{i} s_{j}}$. During the second step, the bonds that were left inactive in the first step can be ignored.

Since the first step does not depend on the signs of the spins, and the probability $p$ is typically quite small, we introduce (following Ref. [7]) a cumulative bond probability. This quantity determines which bond is the next bond to be provisionally activated. The probability that, during the first step, $k-1$ bonds are left inactive and that the $k$ th bond is provisionally activated is equal to

$$
P(k)=(1-p)^{k-1} p .
$$

The cumulative bond probability $C(k)$ is defined as

$$
C(k) \equiv \sum_{n=1}^{k} P(n)
$$

The interval $k-1$ to the next bond to be provisionally activated is obtained by drawing a random number $r$ $(\in[0,1\rangle)$. If this random number lies between $C(k-1)$ and $C(k), k-1$ bonds are skipped and the $k$ th bond is provisionally activated. It is readily seen that this procedure leads to precisely the required probabilities given in Eq. (B2). But the number of operations per spin in the cluster is only of order $p R_{m}^{2}$; near criticality, this quantity is approximately equal to 1 . Thus, the work involved in the decision concerning the actual bond activation is also of order unity.

We check independently whether the resulting probability of activating the first bond at a distance $k$ is equal to that in the conventional approach. Consider a cluster spin, say $s_{0}$, with a chain of neighbors denoted $s_{1}, \ldots, s_{k}$, of which $m$ spins are antiparallel to $s_{0}$ and $l=k-m$ spins are parallel to $s_{0}$, among which $s_{k}$. In the conventional Wolff cluster algorithm, the probability that $s_{k}$ is the first spin to be added to the cluster is, provided that $s_{0}=s_{k}$, given by

$$
1^{m}(1-p)^{l-1} p=(1-p)^{l-1} p .
$$

On the other hand, if we use the cumulative bond probability (B3), this probability is calculated as follows. Either the $k$ th spin is selected directly [if the first random number lies between $C(k-1)$ and $C(k)]$ or one of the $m$ antiparallel spins is selected, say $s_{a}$, which is of course not added to the cluster. In the latter case, another random number is drawn and a new spin is selected. Again, this may be the $k$ th spin, or one of the remaining antiparallel spins between $s_{a}$ and $s_{k}$. Now, let us show that the sum of these probabilities of adding $s_{k}$ as the first spin to the cluster is equal to (B4). Denote the number of selected, "intermediate," antiparallel spins by $i$. There are $\left(\begin{array}{c}m \\ i\end{array}\right)$ possibilities of selecting $i$ intermediate spins. The probability of selecting $s_{k}$ after each of these sequences of spins is $p^{i}(1-p)^{m-i}(1-p)^{l-1} p$. The total probability is the sum over all numbers of intermediate spins

$$
\sum_{i=0}^{m}\left(\begin{array}{c}
m \\
i
\end{array}\right) p^{i}(1-p)^{m-i}(1-p)^{l-1} p=(1-p)^{l-1} p
$$

which is indeed equal to (B4).

As shown in Ref. [7], $C_{j}(k)=1-\exp \left(-\sum_{n=j+1}^{k} K\right)$ $=1-\exp (-[k-j] K)$. By inverting this relation, the bond distance $k$ can be calculated from $C_{j}(k)$, i.e., from the random number. This approach is highly efficient; compared to conventional (Metropolis) algorithms the gain is typically a fac- 
tor $O\left(R_{m}^{d} L^{2}\right)$. Finally we remark that efficient variants of this technique can be applied to long-range $O(n)$ models with $n>1$. Again, bonds are provisionally activated with a probability $p \equiv 1-\exp (-2 K)$; actual activation of a bond be- tween spins $\vec{s}_{i}$ and $\vec{s}_{j}$ is done afterwards with a probability $\left[1-\exp \left(-2 K s_{i, z} s_{j, z}\right)\right] / p$ if $\left(\vec{s}_{i} \cdot \vec{s}_{j}\right)>0$ (where $z$ defines the spin-flip direction of the cluster-formation step) and otherwise with probability 0 .
[1] V.L. Ginzburg, Fiz. Tverd. Tela 2, 2031 (1960) [Sov. Phys. Solid State 2, 1824 (1960)].

[2] H.P. Deutsch and K. Binder, J. Phys. II (France) 3, 1049 (1993).

[3] M.E. Fisher, Rev. Mod. Phys. 46, 597 (1974).

[4] K.K. Mon and K. Binder, Phys. Rev. E 48, 2498 (1993).

[5] P.A. Rikvold, B.M. Gorman, and M.A. Novotny, Phys. Rev. E 47, 1474 (1993).

[6] E. Luijten and H.W.J. Blöte, Phys. Rev. Lett. 76, 1557 (1996); 76, 3662 (1996).

[7] E. Luijten and H.W.J. Blöte, Int. J. Mod. Phys. C 6, 359 (1995).

[8] M.E. Fisher, S.-k. Ma, and B.G. Nickel, Phys. Rev. Lett. 29, 917 (1972)

[9] J. Sak, Phys. Rev. B 8, 281 (1973).

[10] M.E. Fisher, in Proceedings of the Summer School on Critical
Phenomena, Stellenbosch, South Africa, 1982, edited by F.J.W. Hahne (Springer, Berlin, 1983).

[11] H.W.J. Blöte, E. Luijten, and J.R. Heringa, J. Phys. A 28, 6289 (1995).

[12] E. Brézin and J. Zinn-Justin, Nucl. Phys. B 257 [FS14], 867 (1985).

[13] S.-k. Ma, Modern Theory of Critical Phenomena (AddisonWesley, Redwood, CA, 1976).

[14] G. Kamieniarz and H.W.J. Blöte, J. Phys. A 26, 201 (1993).

[15] M.P. Nightingale and H.W.J. Blöte, J. Phys. A 15, L33 (1982).

[16] A. Erdélyi et al., Tables of Integral Transforms (McGraw-Hill, New York, 1954), Vol. II.

[17] U. Wolff, Phys. Rev. Lett. 62, 361 (1989).

[18] R.H. Swendsen and J.-S. Wang, Phys. Rev. Lett. 58, 86 (1987). 